\title{
Vitamin D deficiency negatively affects total bone mineral density in adult Brazilian women living in Southern England: baseline results of the D-SOL study
}

\author{
M.M. Mendes ${ }^{1}$, K.H. Hart ${ }^{1}$, P.B. Botelho ${ }^{2}$ and S.A. Lanham-New ${ }^{1}$ \\ ${ }^{1}$ Department of Nutritional Sciences, Faculty of Health and Medical Sciences, University of Surrey, Guildford, Surrey, \\ UK, GU2 $7 X H$ and ${ }^{2}$ Faculty of Nutrition, Federal University of Goiás, GO, Brazil.
}

Vitamin D deficiency, defined as 25-hydroxyvitamin D $(25(\mathrm{OH}) \mathrm{D})$ serum concentrations below 25 nmol/1, may reduce calcium absorption resulting in a defect in bone matrix mineralization that leads to rickets in children and osteomalacia/osteoporosis in adults. Low 25(OH)D serum concentrations have been associated with low bone mineral density and risk of fractures ${ }^{(1)}$. The D-SOL study (A Systems Biology Approach to the Interaction between Vitamin D Supplementation and Sunlight Exposure in Brazilian Women living in Opposite Latitudes) involves two parallel vitamin D randomised controlled trials, one held in the UK and the other in Brazil, with identical study designs.

This cross sectional sub-analysis aimed to investigate the association between vitamin D status and bone health parameters in Brazilian women living in Southern England and included 51 Brazilian women aged 20-59 years recruited for the D-SOL UK RCT arm in December 2016. Radial bone geometry and density were measured using peripheral quantitative computed tomography (pQCT) at diaphyseal $(66 \%)$ and distal $(4 \%)$ sites. Serum $25(\mathrm{OH}) \mathrm{D}$ concentration was measured by liquid chromatography tandem mass spectrometry.

At baseline, mean vitamin D status was $35.12 \pm 15.11 \mathrm{nmol} / 1$, with $27.5 \%$ of the women below the vitamin D deficiency cut-off of $25 \mathrm{nmol} / \mathrm{l}^{(2)}$. A total of $54.9 \%$ of the women had vitamin D levels below $50 \mathrm{nmol} / \mathrm{l}$, which is defined as 'vitamin D inadequacy' by the US Institute of Medicine ${ }^{(3)}$. Women with vitamin D deficiency had significantly lower total volumetric bone mineral density (vBMD) $(p=0.015)$ and cortical vBMD $(p=0.03)$ at the diaphyseal site than those presenting vitamin $\mathrm{D}$ inadequacy. Deficient women also showed significantly lower total vBMD at the diaphyseal site than those with levels above $50 \mathrm{nmol} / 1(\mathrm{p}=0 \cdot 047)$. There were no significant differences between women with inadequate levels and those with levels above $50 \mathrm{nmol} / 1$, for any of the bone measurements. After controlling for weight and age, these differences persisted for total vBMD (ANCOVA $p=0 \cdot 039$ ), but not for cortical vBMD $(\mathrm{p}=0.145)$. There were no significant differences in other $\mathrm{pQCT}$ measurements between the three vitamin $\mathrm{D}$ status groups.

This study has shown that women with serum 25(OH)D concentrations below $25 \mathrm{nmol} / \mathrm{l}$ have poorer total vBMD than those with higher levels, being in accordance with the most accepted minimum threshold for vitamin D levels of $25 \mathrm{nmol} / 1$ to prevent detrimental effects to bone health, with findings independent of body size. The specific nutrient:gene/endocrine interactions and how these concomitantly affect vitamin D metabolism and bone health indices will be explored further within each country cohort. The D-SOL Study will provide valuable insights into the specific predictors and requirements to prevent vitamin D deficiency for Brazilian women living abroad and in their native country.

MMM gratefully acknowledges funding from the Science Without Borders Scheme (CNPq).

1. Lips P \& van Schoor NM (2011) Best Pract Res Clin Endocrinol Metab, 25, 585-91.

2. Scientific Advisory Committee on Nutrition (2016) Vitamin D and Health. Public Health England.

3. Institute of Medicine (2011) Dietary Reference Intakes for Calcium and Vitamin D. Washington (DC): National Academic Press. 